\title{
RECOGNITION THE DROPLETS IN GRAY SCALE IMAGES OF DROPWISE CONDENSATION ON PILLARED SURFACES
}

\author{
Helene Martin ${ }^{1}$, SolmazBoroomandiBarati ${ }^{2, *}$, Jean-Charles Pinoli ${ }^{1}$, \\ StephaneValette ${ }^{3}$ and Yann Gavet ${ }^{1}$ \\ ${ }^{1}$ Ecole Nationale Superieure des Mines de Saint-Etienne, \\ LGF UMR CNRS 5307, SAINT-ETIENNE, France \\ ${ }^{2}$ Univ Lyon, Ecole Nationale Superieure des Mines de Saint-Etienne, \\ LGF UMR CNRS 5307, SAINT-ETIENNE, France \\ ${ }^{3}$ Univ Lyon, Ecole Centrale de Lyon, LTDS UMR CNRS 5513, F-69134, \\ LYON, France
}

\begin{abstract}
This study deals with developing an image processing algorithm that is able to recognize spherical and ellipsoidal droplets growing on pillared surfaces during heterogonous dropwise condensation. The problem with recognizing droplets on the pillared substrates is that droplets are very similar to the pillars or they cover several pillars at the same time, so characterizing the pillars is very important. On the other hand the droplets are not always perfectly spherical or they are connected and form irregular shapes, in such cases the ability to recognizing and separating connected droplets is another challenging step. The method that is used here consists of three main parts: pillars characterization, droplets categorizing and droplets segmentation. The result of this algorithm will be binarized images that enable to extract the information related to the size and density of droplets needed for studying droplets evolution during time. Also a computer simulation method is proposed to generate ellipsoidal droplets on pillared substrate. The results of this algorithm then are validated by comparing with results from experimental procedure.
\end{abstract}

\section{KEYWORDS}

Dropwise Condensation, Image Segmentation, Textured Surface, Droplets Recognition

\section{INTRODUCTION}

Dropwise condensation has become an attractive process during last decades thanking to its higher heat transfer coefficient (about 5 to 7 times)compared to filmwise condensation [1]. Generally dropwise condensation includes five main steps: nucleation of initial droplets, growth due to adsorption, growth due to coalescence, nucleation of new droplets, and sliding of very big drops.

Natarajan Meghanathan et al. (Eds) : ICCSEA, WiMoA, SPPR, GridCom, CSIA - 2017

pp. 115-126, 2017. (C) CS \& IT-CSCP 2017

DOI : $10.5121 /$ csit.2017.71109 
Nucleation step can occur homogenously, when there is no preference between different points to grow the droplets, and heterogeneously otherwise. In the process of dropwise condensation, at first small droplets nucleate all around the surface, then these small droplets start to grow by adsorbing water molecules from humid air. After awhile if two or more droplets become big enough to overlap, they will merge and form a bigger droplet -called daughter droplet- in the mass center of the parents [2]. This phenomenon is called coalescence in literature. Although coalescence is a mass conservative process, the area covered by daughter droplet is lower than the summation of area covered by its parents. This will lead to forming vacant area around daughter droplet, in which new small droplets can nucleate and grow. Both these steps will change number of droplets per unit area $\left(N_{t}\right)$ during time and lead to a temporal distribution [3].

Up to know the process of dropwise condensation was mostly studied on flat surfaces that considered as homogenous process [4,5] or on the surfaces with coatings for heterogeneous nucleation [6]. But since the chemical coatings have harmful effects on environment, heterogeneous dropwise condensation on textured surfaces is now more attractive to scientists. Using textured surfaces also enabled us to control droplets configuration on the surface [7]. The problem with condensation on textured surfaces is extracting process information like the radius and density of droplets, especially when they really are similar to the surface texturations. Also the shape of droplets is not perfectly circular in latest steps.

There are lots of methods for droplets recognition in the gray scale images on flat surface. The most adapted methods to recognize spherical droplets in gray scale images are Hough transform [8] and its improvements, such as the normal-line Hough transform [9] and the coherent circle Hough transform [10] methods. Muthukrishnan and Radha [11] used an algorithms based on edge analysis and generated the images by an edge detection method. This method can also be used for droplets that are not perfectly circular. There are also another types of algorithms that use the gray-level intensities as a drop presence indicator. These algorithms use the gray-level intensities directly like the PIV methods [16] and watershed [17] or indirectly like the appearance-based approaches [18]. But the problem with these algorithms on pillared surfaces is the similarity between droplets and pillars and the overlapping droplets. This is because on pillared surfaces the droplets are canalized and form non-circular connected droplets between the pillars.

This study aims to develop an image processing algorithm for recognizing different sizes of droplets on pillared surfaces. This algorithm is able to recognize pillars from droplets or the connected droplets that are not perfectly circular. On the other hand a method for simulating ellipsoidal droplets is proposed that is more suitable for dropwise condensation on textured surfaces.

\section{EXPERIMENTAL APPARATUS}

Water droplets are formed on the poly carbonate surface of $1 \mathrm{Cm} \times 1 \mathrm{Cm}$ with temperature of around $17^{\circ} \mathrm{C}$ in contact with humid hot vapor, that is chosen $1{ }^{\circ} \mathrm{C}$ below the dew point of humid air in order to start nucleation of small droplets. Vapor temperature is set at $30^{\circ} \mathrm{Cwith}$ relative humidity of $40 \%$ that is maintained by a compressor outside the chamber. The substrate is textured by pillars with diameter of $12.5 \mu \mathrm{m}$ and height of $3 \mu \mathrm{m}$ that are positioned in the distance of $50 \mu \mathrm{m}$. High resolution CCD camera is used to record nucleation and growth of droplets in time interval of $1 \mathrm{~s}$. The images taken by CCD camera then are binarized and used to 
extract the information of droplets size and number at each time step to validate the results of simulation. The initial density and size of droplets are $3.7 \times 10^{7} \mathrm{~m}^{-2}$ and $6 \times 10^{-6} \pm 1 \times 10^{-6} \mathrm{~m}$ respectively.

\section{IMAGE PROCESSING}

The aim of this section is binarizing the images of real substrate in order to extract the data related to size and number of droplets at each time step. The schematic diagram of the algorithm used to binarize the gray scale images is presented in figure (1).

The problem with pillared surfaces is that pillars are very similar to droplets because of their spherical shape and also because in gray scale images both pillars and droplets exhibit darker boundaries and brighter centers. So the first step will be pillar characterizing in order to separate them from droplets. In this regard, the first image of the considering set is used because the drop presence can be neglected. Then since the images are taken without shifting, one can consider the same pillars for other images. As pillars are perfectly circular with known radius, the circle Hough transform is the most adapted technique. This method consists of implementing an accumulator array which refers to the circle center position probability image for a given radius. For this purpose, an edge detection operator is firstly applied to the original image to get a binary edge image. Then, for each edge point, a circle with this center and the given radius is incremented to the accumulator array. This circle corresponds to the possible center locations of the circle passing through this edge point. Consequently, the peaks of the accumulator array correspond to the most probable center locations of the circles in the images. This principle is used for a range of radius to limit information loss. In our case, as we know the real size of pillars (between 10 and $20 \mu \mathrm{m}$ ), the radius range considered for the Hough transform is [10 - 30] pixels, according to the camera magnification. It is important to consider a quite narrow range in order to get accurate results and a low computational time. To improve again the algorithm performances, the coherent Hough transform is used.

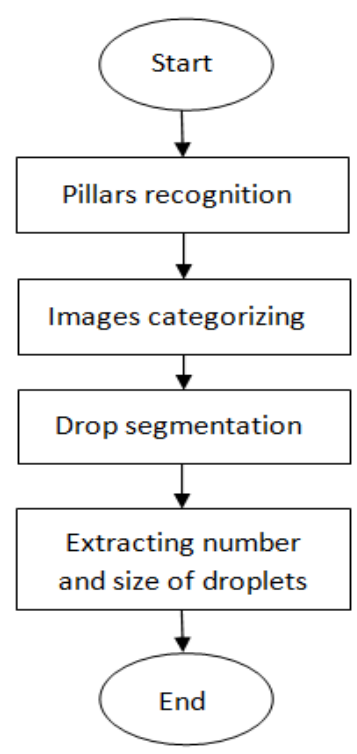

Figure (1): Schematic diagram of image processing algorithm 
In the second step the images are divide in to images that containing very small, small, medium and big drops. The first two groups refer to the droplets that grow by adsorption. The third group corresponds to the coalescence step and the last one corresponds to the steady state, where the changes in size and number of droplets are negligible. After this categorization, the corresponding image processing algorithm is applied to each group. In this regard, firstly, ten percent of images have been chosen regularly in order to reduce the computation time. Since the time step between images is $1 \mathrm{~s}, 10 \%$ of images means selecting one image at each 10 seconds. Then the corresponding gray-tone level histograms of each image are determined as shown in Figure (2). The number of major peaks in each histogram varies as a function of time that represents the different sizes of droplets. Thus, the size ranges of each group are characterized as:
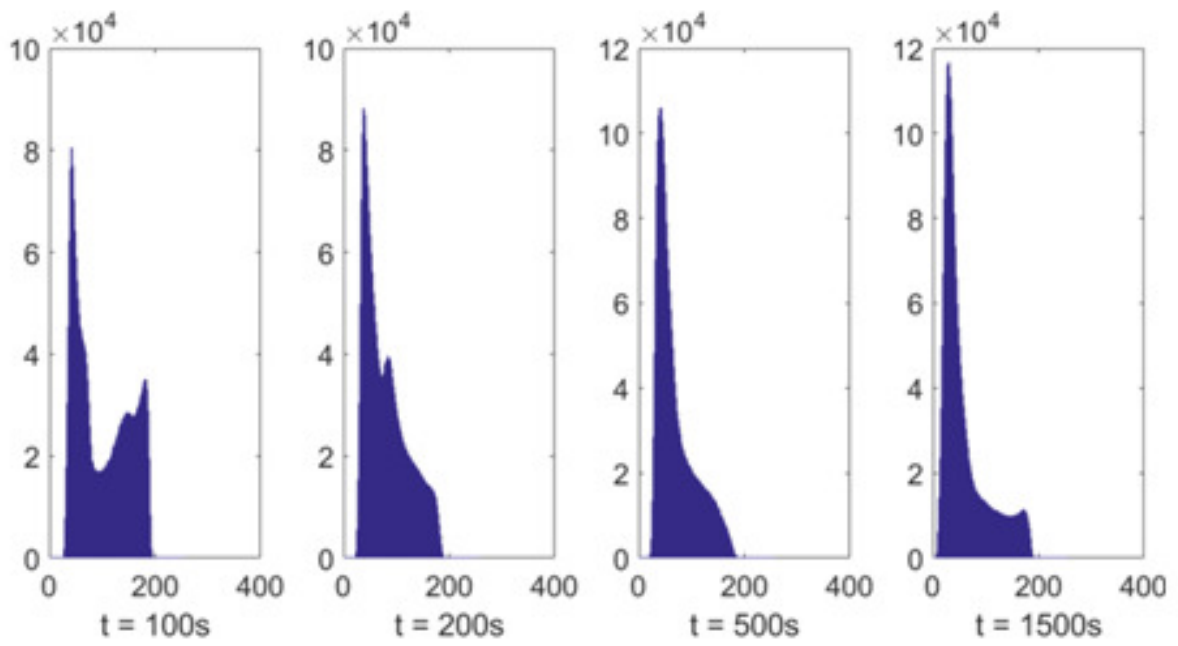

Figure (2): Gray-tone histogram of images taken at $\mathrm{t}=100,200,500$, and 1500s. The histograms from left to right represent very small, small, medium, and big droplets.

- Very small drops: image with three major peaks representing background, the drop edge and the drop center.

- Small drops: image with two major peaks. In this group the peak corresponds to background is removed because drops tend to cover entirely the sample.

- Medium drops: following the process the drops start to coalesce and their area becomes larger with the same intensity as background. So histogram will show one peak.

- Big drops: finally gray tone histogram shows two major peaks again related to the drop edges and the drop centers. This step corresponds to forming big droplets.

Figure (3) illustrates these four droplets groups. According to this figure the first two groups of droplets are perfectly circular and smaller than the pillars. Also they appear with darker points on the surface that are recognizable. While medium and big droplets tend to have more ellipsoidal shapes with center points very similar to the surface of background. So recognizing these two latest groups needs another additional step to separate droplets from the background. 


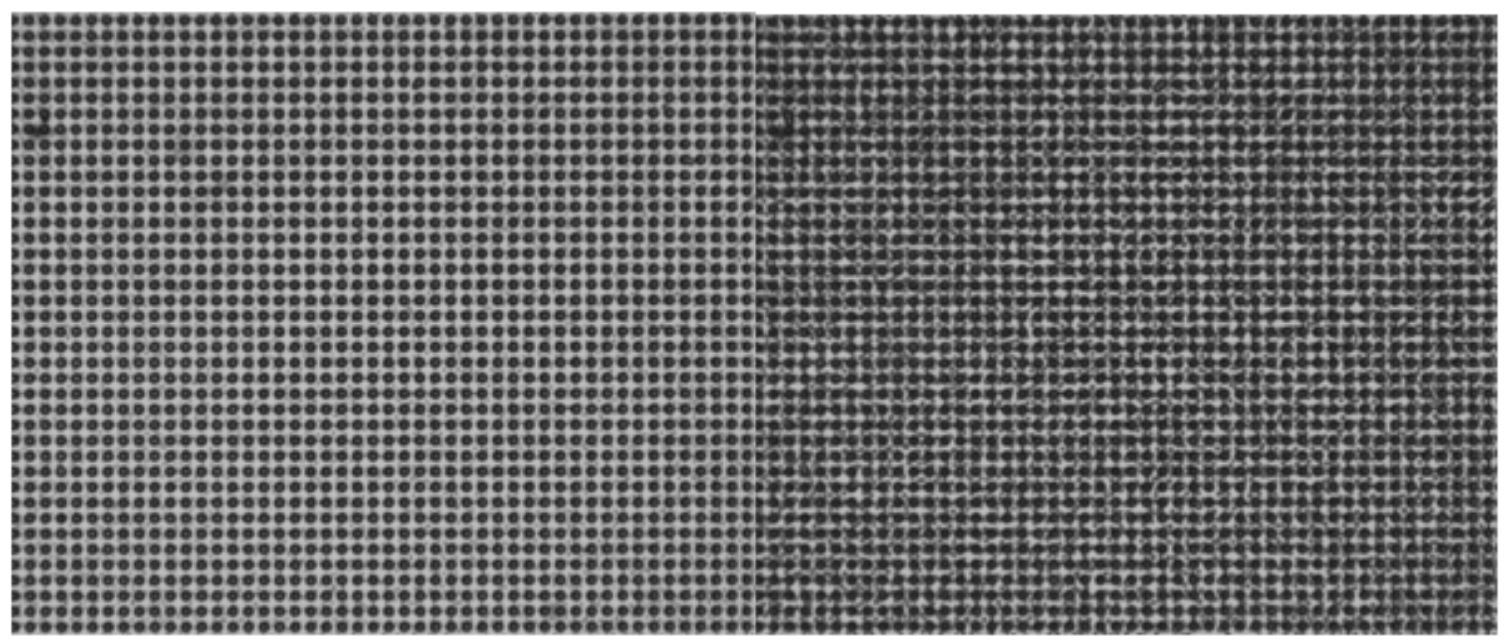

(a)

(b)

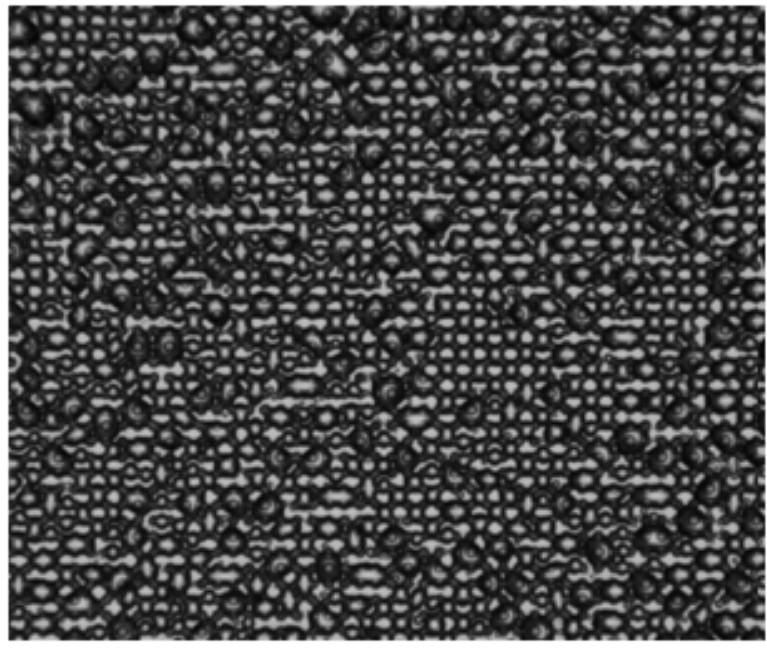

(c)

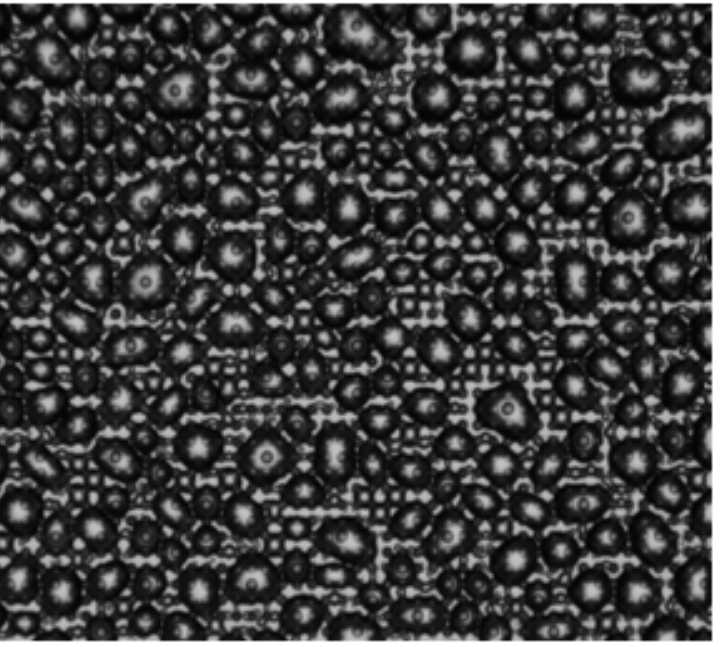

(d)

Figure (3): Illustrations of (a) very small droplets, (b) small droplets, (c) medium droplets, and (d) big droplets.

Binarization of each group of droplets then is done with different methods:

\subsection{Very small drops:}

Very small droplets are appeared in the images as very small dots between the pillars (figure $(3, a))$. At first, in order to ignoring the pillars, they are removed by means of the method describing in the pillar characterization step. Thus, a gray-level image is obtained with black holes at the pillars places. By comparing the gray tone histograms of very small droplets and the gray tone histogram of image without the droplets, it reveals that the histogram of the first image is less spread than the histogram corresponds to very small droplets. This comparison validates that since the drops are darker than substrate, they modify gray-level intensities of the image towards the vertical axis. The idea is that if we draw the two histograms in the same diagram, the first non-zero point, at which they meet or the non-zero superposition point of these two histograms can be accounted as the thresholding value for gray scale image of very small 
droplets. Then, labeling the regions on the tresholded images enable us to have the number of droplets that is equal to the number of regions. Finally mean radius of droplets can be calculated from equation (1) by knowing the total area of droplets $\left(A_{t o t}\right)$ :

$\mathrm{R}_{\mathrm{ave}}=\sqrt{\frac{A_{t o t}}{N \pi}}$

Equation (1)

\subsection{Small drops:}

When droplets grow du to adsorption they are perfectly circular (figure $(3, b)$ ), so they can be recognized by Hough transform. In the binary image that is obtained after eliminating the pillars, $A_{\text {tot }}$ can be calculated, so in order to get the number of drops, it is just needed to separate the overlapping droplets. For this purpose, the binary image shown in figure $(4, a)$ is turned into a distance map using the Euclidean distance as is shown in figure $(4, b)$. The distance map shows the points inside the droplets with maximum distance from the background area. Therefore, for two overlapping droplets the distance map will eliminate the area of intersection because it is close to background and show two separate points related to droplets centers. In other words, the distance map shows just the droplets centers separately and can be used as an efficient tool for separating overlapping droplets. Now the watershed technique can be applied to binarize the gray scale image. A labeling step enables to get the drop number and finally, equation (1) gives the mean radius of drops.

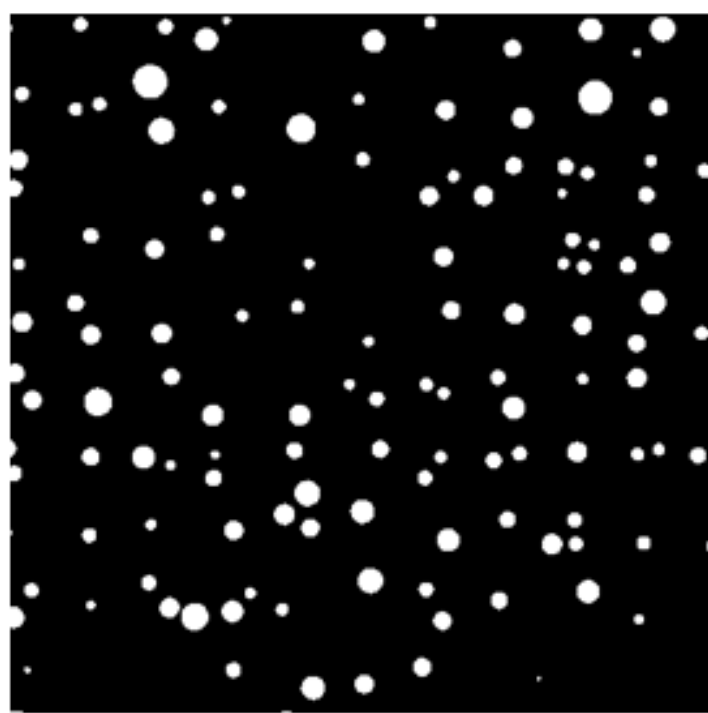

(a)

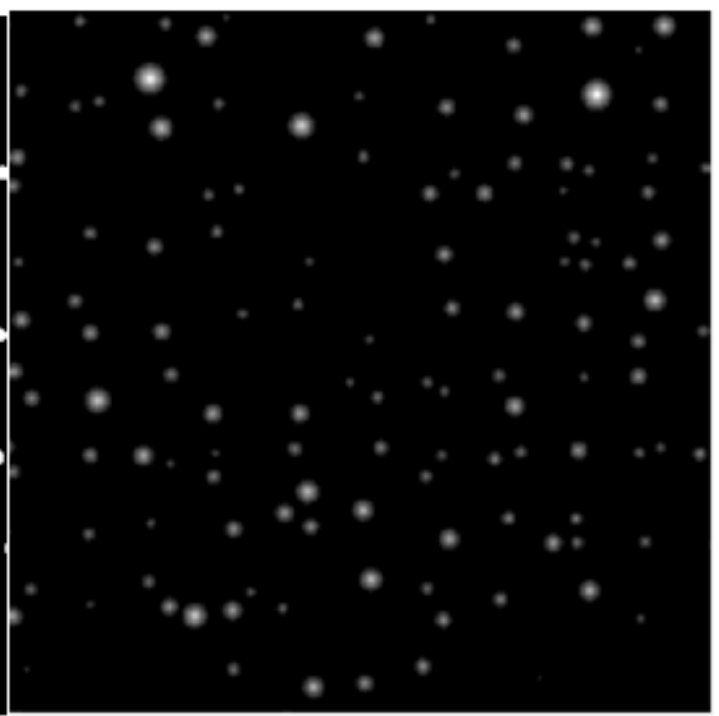

(b)

Figure (4): (a)Binary image of small drops, (b) Distance map of small drops. The distance map can separate the overlapping droplets by emphasizing on the points that are in longer distance from contours of droplets. 


\subsection{Medium drops:}

Medium drops form a continuous cluster around the pillars. The problem with these droplets is that their centers are very similar to the background region (Figure $(3, \mathrm{c}))$. So, at first the drops centers should be determined by some criteria. The droplets center $\mathrm{s}$ are different from background region from two points of view, they are more convex, but they have more spread gradient magnitude. Therefore, the original images are firstly thresholded by Otsu's method to get brighter regions. Then two techniques are applied to sufficiently large white regions. The regions that correspond to a convexity rate under $4 \%$ figure $(5, a)$ or the ones which correspond to a low rate of gradient magnitude according to figure $(5, \mathrm{~b})$ are related to drops centers. The limited rate of gradient magnitude corresponds to $4 / 5$ of the mean gradient magnitude of the neighborhoods. Finally, the binary images are made by applying the watershed method.

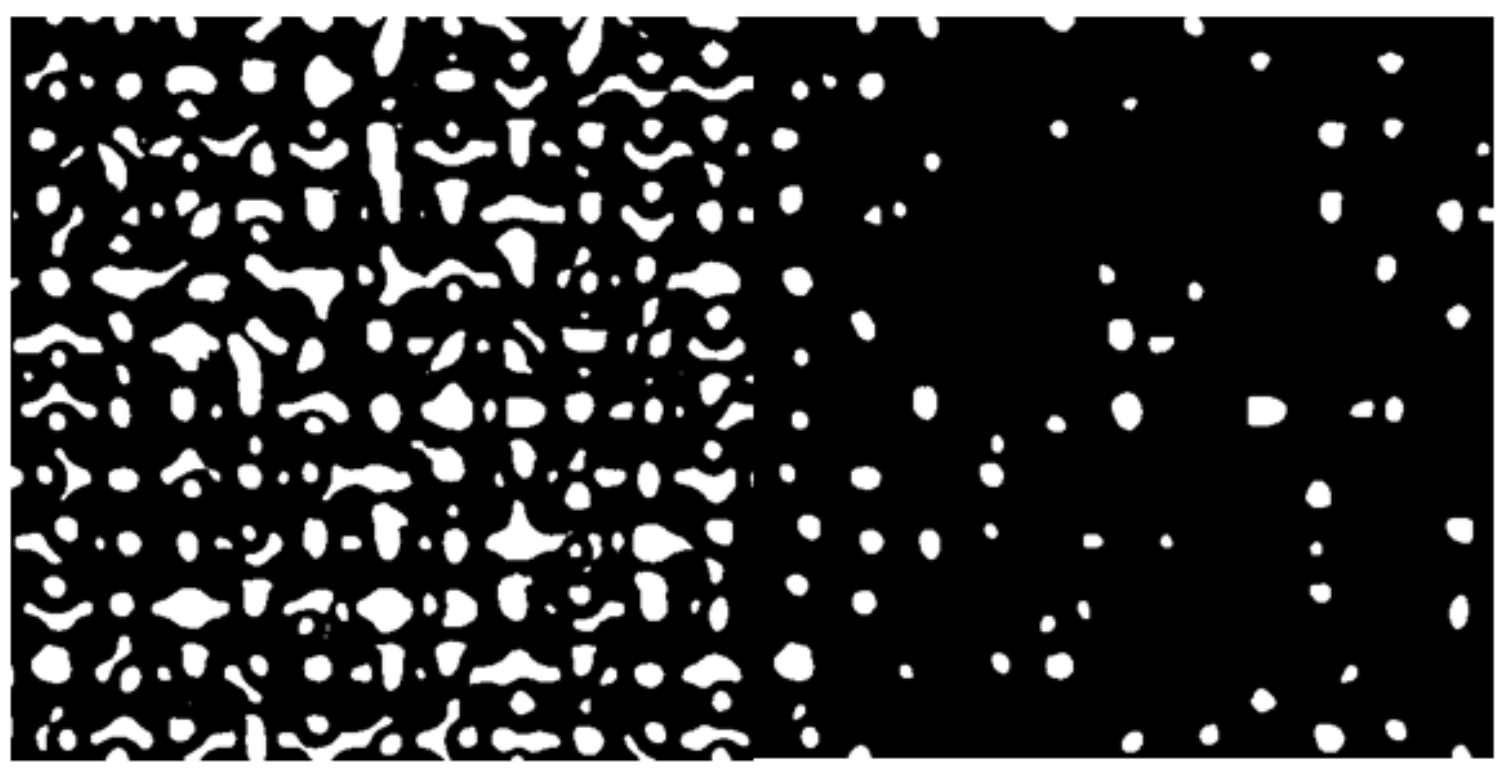

(a)

(b)

Figure (5): (a) Low gradient part of medium drops, (b) Convex part of medium drops

\subsection{Big drops:}

Big drops can cover entirely several pillars as shown in Figure $(3, d)$. For binarizing such images, shrinking and gradient properties must be applied. At first, the images are thresholded by Otsu's method, and then the black parts are shrunk in order to get their skeletons around the white regions, which correspond to either drops centers or the background. As was explained for the medium drops, the average of gradient magnitude is calculated on each white region and then. The distribution of this value shows two main peaks related to intensity of background and intensity of droplets centers. The median between these two peaks can be used as an tresholding value for gray scale image. However, when several white regions belong to the same drop, the watershed technique cannot be applied directly. To solve this problem, the white regions are dilated and overlaid with the pillars image. Thus, the white regions which belong to the same drop are connected, then applying a test on convexity enables to reconstruct each drop center. 
Finally, a watershed technique is used to detect each drop region that enables to get the drops number and mean radius.

\section{AlgORITHM FOR SimUlating DROPLETS NUCLEATION AND GROWTH}

As was explained before, the medium and big droplets are not perfectly spherical and they can form several connected ellipses lying between the pillars. So the algorithm that is proposed here, simulates nucleation and growth of ellipsoidal droplets on the pillared surfaces. At first random ellipses with density of $3.7 \times 10^{7} \mathrm{~m}^{-2}$ are distributed on the three dimensional pillared substrate. The height of droplets on the three dimensional substrate is calculated based on their distances with pillars:

1) If center to center distance of droplet and pillar $>$ radius of the pillar, meaning that the droplet nucleates on the surface of substrate, so $\mathrm{z}=0$.

2) If center to center distance of droplet and pillar = radius of the pillar, meaning that the droplet nucleates on the top of the pillars, so $\mathrm{z}=\mathrm{z}_{\text {pillar }}$.

3) If center to center distance of droplet and pillar < radius of the pillar, meaning that the droplet nucleates on the side of the pillars, so $0<\mathrm{z}<\mathrm{z}_{\text {pillar }}$.

Substrate area is considered equal to $1.3 \times 10^{-5} \mathrm{~m}^{2}$, covered by pillars with radius of $25 \mu \mathrm{m}$, height of $10 \mu \mathrm{m}$ and border to border distance of $12.5 \mu \mathrm{m}$. Each ellipse has three unequal radius considered as: $a, b=e . a$, and $d=f . a$ along the axis $X, Y$, and $Z$, where $e$ and $f$ are the random numbers. All the droplets then start to grow thorough an iterating loop. At each iteration, there is an inner loop in which the droplets that are in touch with pillars are recognized, because if a droplet touches a pillar, they will stay in touch until the end of the process and this will be important during coalescence step. After this inner loop, each droplet grows by adsorbing water molecules from humid air, then the droplets that are in touch in each of the planes $X-Y, X-Z$, or $Y$ $Z$ unite and form a bigger droplet called daughter drop. The position of daughter drop is identified as follow:

1) If both or none of the parents touch at least one pillar, the daughter drop will be in the mass center of its parents.

2) If just one of the parents touches a pillar, then the daughter drop will locate at its center point.

The result of coalescence is producing more vacant area on the substrate, on which the small ellipses can nucleate at each iteration. Finally, if a droplet is big enough to slide from the surface, it will leave the surface and clean off other droplets on its path.

This algorithm was written in Matlab environment and took 2 hours for printing the final results. 


\section{RESULTS AND DISCUSSION}

Figures (6)and (7) compare the results of droplets density $\left(N_{t}\right)$ and mean radius $\left(r_{\text {ave }}\right)$ between real images and droplets generated during the computer simulation. As it can be seen from these images, at first the surface is covered by huge amount of very small droplets. After a while the droplets start to grow by adsorbing water molecules and coalescence, this will result in rapid changes in the graphs of $r_{a v e}$ and $N_{t}$. This phase relates to small and medium droplets. In this step the shape of droplets changed from circular to more ellipsoidal drops. By increasing the size of droplets, new small droplets start to nucleate in the vacant area produced during coalescence. These small droplets will balance the number and average size of droplets as well as percentage of area occupied by the droplets. Therefore in the final stages, an approximately constant pattern in the graphs of $r_{\text {ave }}$ and $N_{t}$ is visible.

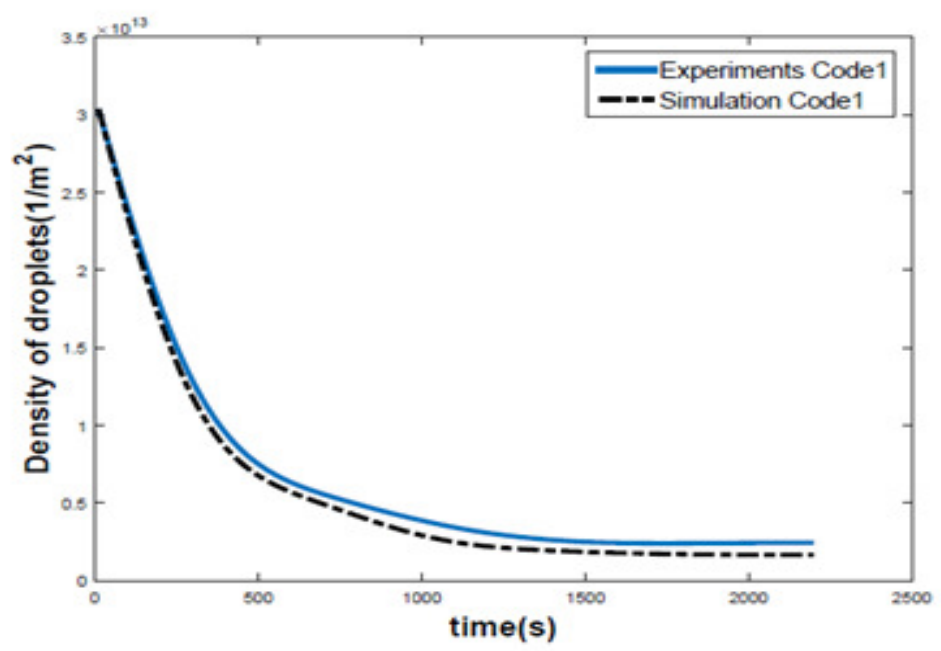

Figure (6): Comparison between density of simulated and real droplets

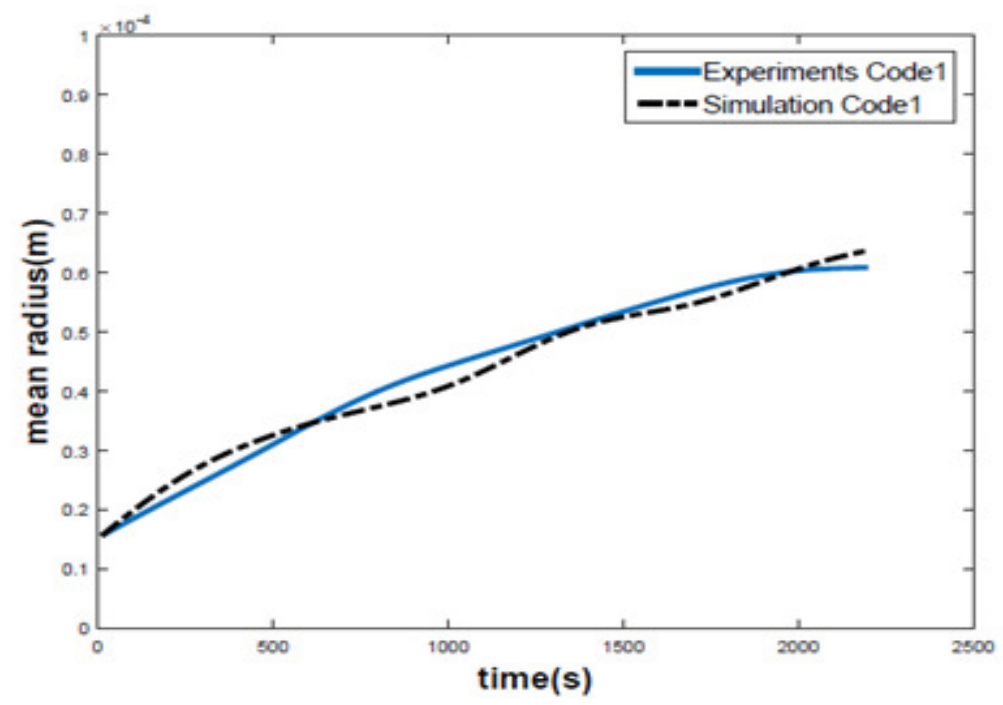

Figure (7): Comparison between mean radius of simulated and real droplets 
Figure (8) shows different stages of ellipsoidal droplets growth including initial nucleation, droplet growth due to adsorption, droplet growth due to coalescence and nucleation of new droplets. Figure $(8, a)$ corresponds to nucleation of initial droplets that are introduced as very small droplets, in this earlier stages the percentage of surface covered by liquid is very small and the images are more black. The initial droplets are more circular and the their radius $a, b$, and $d$ are almost the same. Then these droplets start to grow by adsorbing water molecules from humid air and turn into small droplets that are shown in Figure $(8, b)$. Medium and big droplets (Figure $(8, c)$ and $(8, d))$ are droplets that grow mainly due to coalescence and consequently the area occupied by droplets increases significantly. These droplets that are more ellipsoidal are able to cover the area of several pillars.

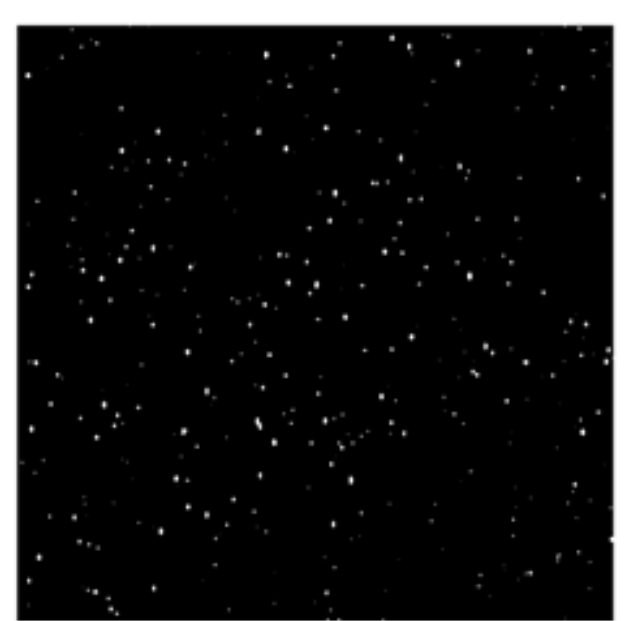

(a)

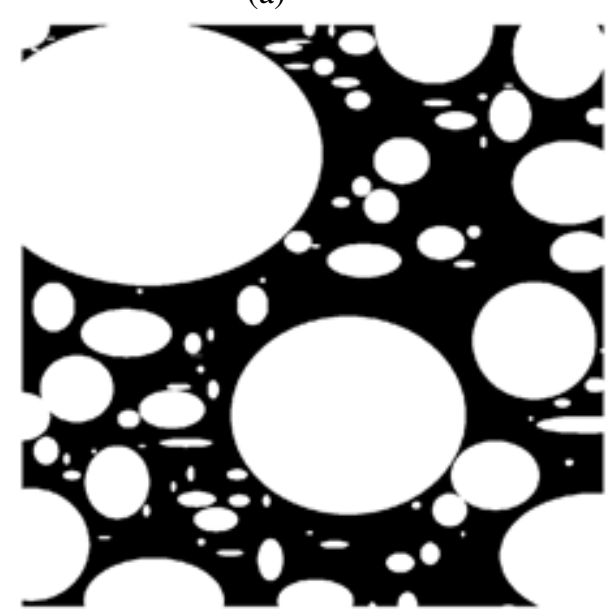

(c)

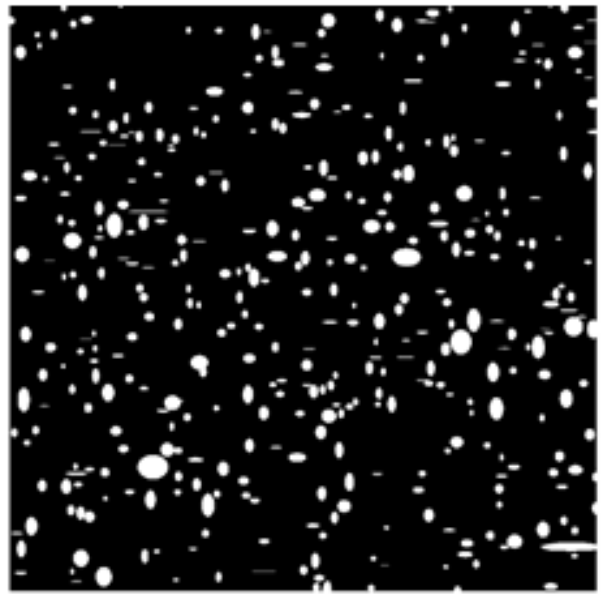

(b)

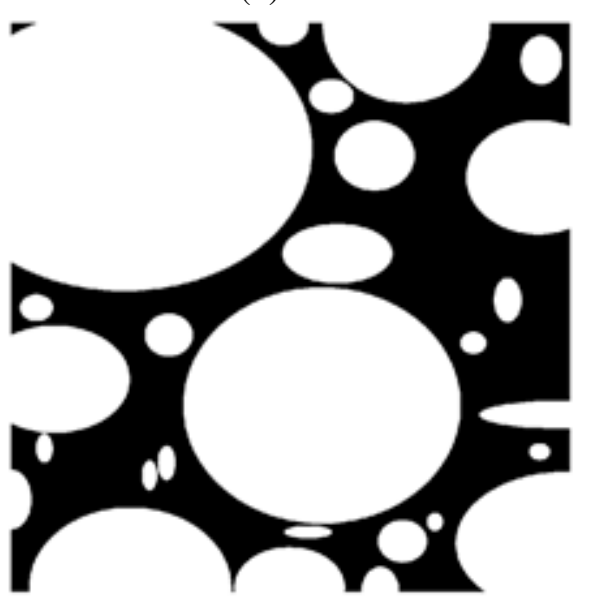

(d)

Figure (8): Different stages of ellipsoidal droplets growth. (a) distribution of initial droplets (b) droplets growth due to adsorption, (c) nucleation of new small droplets in the vacant area of the substrate, and (d) droplets growth due to coalescence. 


\section{CONCLUSION}

In this research we developed an image segmentation method for extracting radius and density of droplets growing on a textured surface from gray scale images. The method divides droplets in to four groups based on their radius and apply different segmentation methods for each group. Then the binarized images produced with this algorithm are used to calculate radius and density of droplets. An algorithm for generating ellipsoidal droplets on textured surfaces then is proposed that is able to check coalescence of droplets in three dimensions. The results of this algorithm are in acceptable accordance with the data extracted from binarized images.

\section{ACKNOWLEDGEMENTS}

The authors gratefully acknowledge the support of Stephane Valette, Nicolas Pionnier, Remi Berger and Elise Contraires from Ecole Centrale Lyon, who provide the image sets.

\section{REFERENCES}

[1] Baojin Qi, Jinjia Wei, Li Zhang, \& Hong Xu, (2015)“A fractal dropwise condensation heat transfer model including the effects of contact angle and drop size distribution". International Journal of Heat and Mass Transfer, Vol. 83, pp259-272.

[2] Paul Meakin. (1992)“Droplet deposition growth and coalescence”, Reports on Progress in Physics, Vol. 55, No. 2, pp157.

[3] Xue-Hu Ma, Tian-Yi Song, Zhong Lan, \& Tao Bai.(2010) "Transient characteristics of initial droplet size distribution and effect of pressure on evolution of transient condensation on low thermal conductivity surface", International Journal of Thermal Sciences, Vol.49, No. 9, pp1517-1526.

[4] Maofei Mei , Feng Hu, \& Chong Han , Yanhai, (2015) "Time-averaged droplet size distribution in steady-state dropwise condensation", Cheng International Journal of Heat and Mass Transfer,Vol. 88, pp 338-345.

[5] leon r. glicksman \& andrew w. hunt, JR , (1972) "Numerical simulation of dropwise condensation" ,International Journal of Heat and Mass Transfer, Vol. 15, pp. 2251-2269.

[6] S. Vemuri \& K.J. Kim, (2006) "An experimental and theoretical study on the concept of dropwise condensation”, International Journal of Heat and Mass Transfer, Vol.49, pp649-657.

[7] Basant Singh Sikarwar, Sameer Khandekar \& K. Muralidhar. (2012),"Mathematical modelling of dropwise condensation on textured surfaces", Sadhana, Vol.38, No.6, pp1135-1171.

[8] Soo-Chang Pei \& Ji-Hwei Horng. (1995), "Circular arc detection based onhough transform”, Pattern recognition letters, Vol.16, No.6, pp615-625.

[9] Xiaoran Yu, Dongchang Xing, Tatsuya Hazuku, Tomoji Takamasa, Takaski Ishimaru, Yuji Tanaka, and TatsuroAkiba. (2009),"Measurement technique for solid-liquid two-phase flow using normal-line hough transform method". In Journal of Physics: Conference Series,Vol.147, pp 012053. IOP Publishing.

[10] T. J. Atherthon and D. J. Kerbyson. (1993), "Using phase to represent radius in the coherent circle hough transform", In Hough Transforms, IEEColloquium on, pp5-1. IET. 
[11] R. Muthukrishnan and M. Radha. (2011), "Edge detection technique for image segmentation", International journal of recent trends in engineering, Vol.1, No.2, pp250-254.

[12] R. Lindken and W. Merzkirch. (2002), "A novel piv technique for measurements in multiphase flows and its application to two-phase bubbly flows",Experiments in fluids, Vol.33, No.6, pp814-825.

[13] S. Beucher and F. Meyer. (1990), "The morphological approach to segmentation; the watershed transformation", Optical Engineering-New York-Marcel Dekker Incorporated, Vol.34, pp433-433.

[14] X. Zabulis, M. Papara, A. Chatziargyriou, and T. D. Karapantsios. (2007), "Detection of densely dispersed spherical bubbles in digital images based in template matching technique - application to wet foams", Physicochemical and Engineering Aspects,Vol.309, No.1, pp96-106. 